Article

\title{
Thermodynamic Black Holes
}

\author{
George Ruppeiner \\ Division of Natural Sciences, New College of Florida, 5800 Bay Shore Road, Sarasota, FL 34243, USA; \\ ruppeiner@ncf.edu
}

Received: 10 April 2018; Accepted: 11 June 2018; Published: 13 June 2018

\begin{abstract}
Black holes pose great difficulties for theory since gravity and quantum theory must be combined in some as yet unknown way. An additional difficulty is that detailed black hole observational data to guide theorists is lacking. In this paper, I sidestep the difficulties of combining gravity and quantum theory by employing black hole thermodynamics augmented by ideas from the information geometry of thermodynamics. I propose a purely thermodynamic agenda for choosing correct candidate black hole thermodynamic scaled equations of state, parameterized by two exponents. These two adjustable exponents may be set to accommodate additional black hole information, either from astrophysical observations or from some microscopic theory, such as string theory. My approach assumes implicitly that the as yet unknown microscopic black hole constituents have strong effective interactions between them, of a type found in critical phenomena. In this picture, the details of the microscopic interaction forces are not important, and the essential macroscopic picture emerges from general assumptions about the number of independent thermodynamic variables, types of critical points, boundary conditions, and analyticity. I use the simple Kerr and Reissner-Nordström black holes for guidance, and find candidate equations of state that embody several the features of these purely gravitational models. My approach may offer a productive new way to select black hole thermodynamic equations of state representing both gravitational and quantum properties.
\end{abstract}

Keywords: black hole thermodynamics; information geometry of thermodynamics; thermodynamic curvature; Kerr black hole; Reissner-Nordström black hole; critical phenomena

\section{Introduction}

Thermodynamics rests on general principles spanning a wide range of physical systems, from pure fluids to black holes. I focus here on the theory of black holes that has been brought into thermodynamics via black hole thermodynamics (BHT) [1]. The idea contributed in this paper is that there is basic thermodynamic information to be found by considering the interplay between macroscopic and mesoscopic size scales in a system. This viewpoint is applied by linking the thermodynamic curvature $R$ with the free energy $\phi$ from the theory of critical phenomena. Applications in conventional thermodynamic have yielded strong results. We might reasonably expect this method to extend to the black hole scenario.

In the conventional thermodynamic scenario, thermodynamic fluctuation theory allows us to probe mesoscopic size scales using macroscopic information [2,3]. Perhaps not as well known is that the theory of critical phenomena, via hyperscaling, allows us to reverse the tables, and use mesoscopic properties to illuminate the macroscopic thermodynamics [4]. These complementary ideas are combined here to get a differential equation for the free energy by using the thermodynamic Ricci curvature scalar $R$ from the thermodynamic information geometry. Assuming that the free energy follows a scaled form allows us to readily solve for the thermodynamics.

Much work has been done calculating the thermodynamic $R$ from known black hole models; see [5] for a brief recent review. However, this agenda is restricted in terms of its physical impact 
because the models in play typically originate either from general relativity or from string theory. There are good reasons for believing that relativity and quantum mechanics must be combined in some fundamental way in the black hole scenario, and how this is done is not clear. There is no consensus which of several string theory models give the correct physical results. These points are illustrated in Figure 1. The approach in this paper is more active. Instead of passively computing $R$ for models that may be inadequate for representing physical reality, I proceed with model independent thermodynamics.

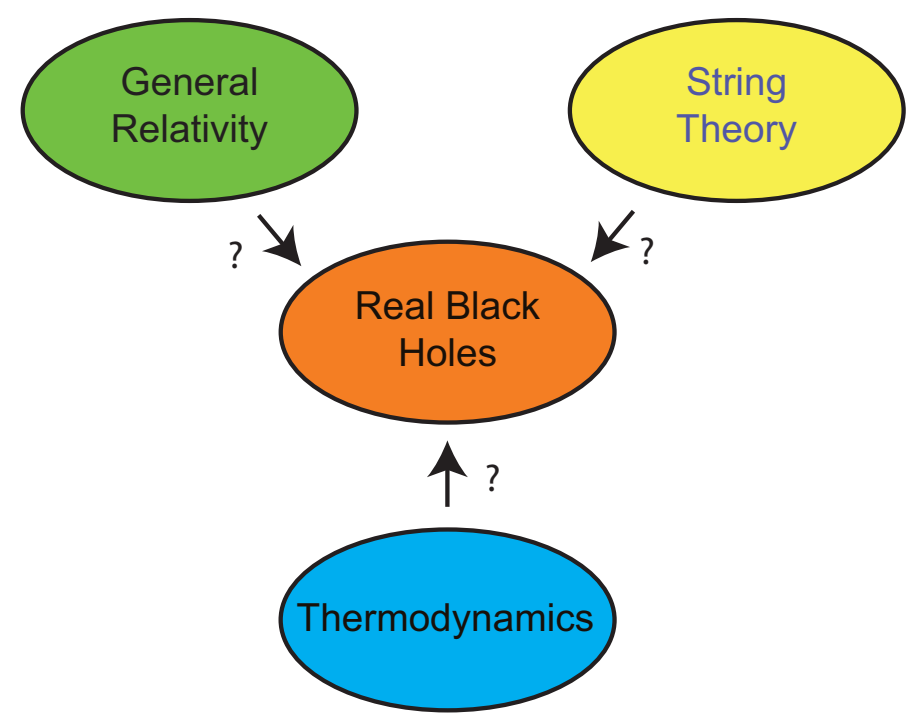

Figure 1. Which theory correctly explains real black holes? General relativity lacks quantum mechanics, and string theory lacks consensus on which model correctly represents physical reality. I propose that a thermodynamic approach might help sort these issues out.

In describing the contribution of the thermodynamic information geometry to black hole thermodynamics, Åman et al. [6] expressed this opinion: "There is a dream, however, which is that [thermodynamic information geometry] may have something to say about the obvious question: what properties must a function have if it is to serve as an entropy function for black holes?" My hope here is that my purely thermodynamic approach offers the opportunity for writing down correct classes of thermodynamic black holes, whose comparison with what is known can offer guidance in the search for a correct theory.

A true understanding of black holes must ultimately be grounded on microscopic models, a topic with a vast literature. A detailed discussion of this theme is beyond the scope of this paper, but I will make a few connections to the literature. A pioneering attempt to employ microscopic state counting to compute the entropy in the black hole scenario was by Strominger and Vafa [7]. Two follow-up efforts were $[8,9]$. Attempts to integrate microscopic models with the geometry of thermodynamics include [10-14]. There are also string theory models based on novel particles, such as R-charges [15], dyons [16], and tidal charges [17]. Ultimately, the structures discussed in this paper must be put into a microscopic context and tested against such results.

\section{Thermodynamic Information Geometry}

In this section, I summarize the thermodynamic information geometry, starting with thermodynamic fluctuation theory.

\subsection{Thermodynamic Fluctuation Theory}

The first element of thermodynamic fluctuation theory consists of the separation of the universe into two distinctive parts, a finite system and an environment tending to infinite size. System and 
environment interact via some boundary across which certain conserved parameters are allowed to fluctuate back and forth. This boundary serves only to separate the two parts, and has zero heat capacity. I consider only cases with two conserved fluctuating variables, but the formalism readily generalizes to more variables. A summary of how thermodynamic fluctuation theory is applied to BHT is given in [18].

Let system and environment each have pairs of fluctuating parameters $\left(X^{1}, X^{2}\right)$ and $\left(X_{e}^{1}, X_{e}^{2}\right)$, respectively. They follow a conservation law

$$
X^{\alpha}+X_{e}^{\alpha}=X_{t o t}^{\alpha}=\text { constant }
$$

with the index $\alpha=1,2$. Figure 2 shows examples of systems, environments, and boundaries in both the pure fluid and the black hole scenarios. I assume that the environments are always very large, and conventional in character. Namely, $\left(X_{e}^{1}, X_{e}^{2}\right)$ and the environmental entropy $S_{e}=S_{e}\left(X_{e}^{1}, X_{e}^{2}\right)$ all scale up in proportion to the environmental size.

In this scenario, it turns out that the only properties of the environment relevant to the fluctuations of the system are the values of two intensive environment parameters

$$
F_{e \alpha}=\frac{\partial S_{e}}{\partial X_{e}^{\alpha}}
$$

around which the fluctuations take place, e.g., temperature, chemical potential ...

(a) $T$

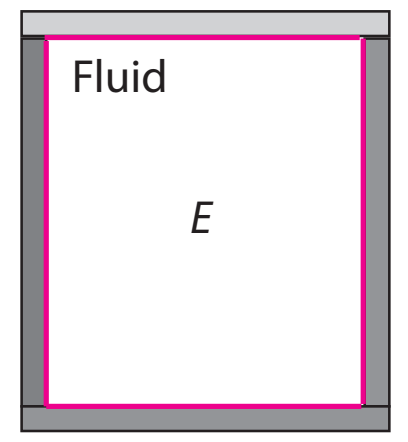

(c) $(T, \mu)$

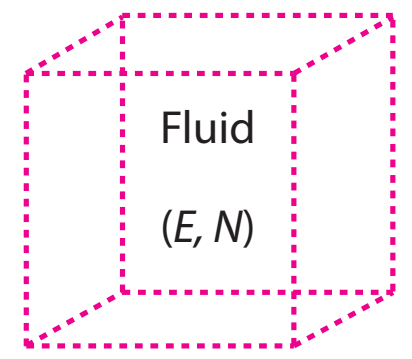

(b) $(T, p)$

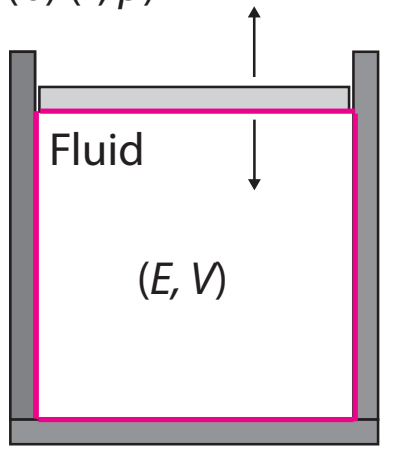

(d) $(T, \Phi)$

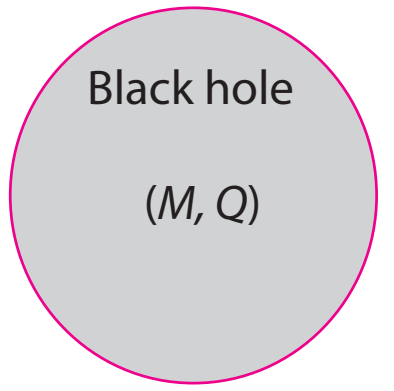

Figure 2. Four thermodynamic systems, environments, and boundaries. Cases of conserved fluctuating variables are: $E$ or $M=$ energy, $V=$ volume, $N=$ number of particles, and $Q=$ charge. Also shown are conjugate nonfluctuating environment parameters: $T=$ temperature, $p=$ pressure, $\mu=$ chemical potential, and $\Phi=$ electric potential. We have: (a) a closed fluid system exchanging heat with its environment; (b) a closed fluid system exchanging heat and volume via a movable piston; (c) an open fluid system with fixed volume exchanging energy and particles; and (d) a Reissner-Nordström black hole exchanging energy and charge via Hawking radiation at the event horizon boundary. 
Let $S=S\left(X^{1}, X^{2}\right)$ denote the entropy of the system. I make no assumption that $S$ depends on its variables in the same way that $S_{e}$ depends on its. Namely, the system is not necessarily a scaled down version of the environment. The only case in Figure 2 where system and environment are necessarily of the same type is Figure 2c, where the boundary is imaginary.

A frequent concern in the literature is the unconventional black hole scaling with system size. For example, combining two identical black holes does not result in a black hole with twice the volume. However, system size scaling does not enter into the thermodynamic fluctuation theory described below. $S=S\left(X^{1}, X^{2}\right)$ is simply taken to be some function of its two variables.

The total entropy of the universe is

$$
S_{\text {tot }}=S\left(X^{1}, X^{2}\right)+S_{e}\left(X_{e}^{1}, X_{e}^{2}\right) .
$$

With fluctuations stable about some environment state $F_{e \alpha}$, the generalized second law of thermodynamics requires that the equilibrium state has a local maximum for $S_{\text {tot }}$. Let $\tilde{X}^{\alpha}$ be the values of $X^{\alpha}$ that maximize $S_{\text {tot }}$ [with the corresponding $\tilde{X}_{e}^{\alpha}$ given by Equation (1)]. Expanding $S_{\text {tot }}$ to first order in

$$
\Delta X^{\alpha}=X^{\alpha}-\tilde{X}^{\alpha}
$$

and using the conservation laws Equation (1), yields

$$
\frac{\partial S}{\partial X^{\alpha}}=\frac{\partial S_{e}}{\partial X_{e}^{\alpha}} .
$$

This pair of algebraic equations equating conjugate variables may be solved for $\tilde{X}^{\alpha}$ if necessary.

Expanding $S_{\text {tot }}$ in Equation (3) to second order about its local maximum, and using Equation (1), yields

$$
\Delta S_{\text {tot }}=\frac{1}{2} \frac{\partial^{2} S}{\partial X^{\mu} \partial X^{v}} \Delta X^{\mu} \Delta X^{v}+\frac{1}{2} \frac{\partial^{2} S_{e}}{\partial X_{e}^{\mu} \partial X_{e}^{v}} \Delta X^{\mu} \Delta X^{v} .
$$

Since both $X_{e}^{\alpha}$ and $S_{e}$ scale up in proportion to the size of the environment (which is assumed to trend to infinity) the second term on the right-hand side of Equation (6) is much smaller than the first, and

$$
\Delta S_{t o t} \simeq \frac{1}{2} \frac{\partial^{2} S}{\partial X^{\mu} \partial X^{v}} \Delta X^{\mu} \Delta X^{v}
$$

Clearly, variations in $S_{\text {tot }}$ depend only on the detailed thermodynamic properties of the system, which are embodied in the Hessian elements of $S$. Effects of the environment appear just through the values of the derivative $F_{e \alpha}$ that set the state about which the system fluctuates, and where the Hessian elements in Equation (7) get evaluated.

Thermodynamic fluctuation theory $[2,3]$ has the probability of the system fluctuation as

$$
P d X^{1} d X^{2} \propto \exp \left(\frac{\Delta S_{t o t}}{k_{B}}\right) d X^{1} d X^{2}
$$

where $k_{B}$ is Boltzmann's constant. Equation (7) now leads to

$$
P \propto \exp \left(-\frac{1}{2} \Delta \ell^{2}\right)
$$

where

$$
\Delta \ell^{2}=-\frac{1}{k_{B}} \frac{\partial^{2} S}{\partial X^{\mu} \partial X^{v}} \Delta X^{\mu} \Delta X^{\nu} .
$$

$\Delta \ell^{2}$ is the line element in the thermodynamic information geometry described in the next subsection. Henceforth, I take $k_{B}=1$, which makes the entropy dimensionless. 
If $\Delta \ell^{2}$ is positive definite, we say that the fluctuations are stable. Necessary and sufficient conditions that $\Delta \ell^{2}$ be positive definite are that

$$
p_{1}=\frac{\partial^{2} S}{\partial\left(X^{1}\right)^{2}}
$$

and

$$
p_{2}=\frac{\partial^{2} S}{\partial\left(X^{1}\right)^{2}} \frac{\partial^{2} S}{\partial\left(X^{2}\right)^{2}}-\left(\frac{\partial^{2} S}{\partial X^{1} \partial X^{2}}\right)^{2}
$$

both be positive.

Thermodynamic stability is frequently lacking in self-gravitating scenarios, because of negative heat capacity. However, the widespread attitude in the literature has been not to worry excessively about this. Åman et al. [19] put it like this: "Nevertheless we believe that the Ruppeiner geometry of black holes is telling us something; our justification is mainly the a posteriori one that once it has been worked out for some examples we will find an interesting pattern." This is certainly my working philosophy in this paper.

Frequent concern is expressed in the literature about the "ensemble dependence" of BHT, but authors are not always clear about what they mean. The phrase "ensemble dependence" is used in ordinary statistical mechanics, where statistical ensembles over microstates are summed to get the thermodynamic properties. For finite systems these sums depend on the character of the boundary between the system and the environment. For example, Figure 2a-c yield the canonical, the Boguslavski, and the grand canonical ensembles, respectively. A central principle of statistical mechanics has the ensembles all yielding equivalent thermodynamic results for infinite systems ("the existence of the thermodynamic limit").

Since black holes are always of finite size, we might think that they must suffer from "ensemble dependence", and that this points to some fundamental conflict with statistical mechanics. However, there is no conflict. First, the general relativity black hole models have no underlying microscopic structures, so there are actually no microscopic statistical ensembles in play there. Second, and perhaps more to the point, in fluctuation theory each boundary between the system and the environment contributes its own character, with each leading to its own distinctive quadratic form in Equation (7). So, for example, the Reissner-Nordström (RN) black hole in Figure $2 \mathrm{~d}$ has an event horizon admitting energy and charge, and the Kerr black hole substitutes angular momentum for charge. These are two different physical problems, and not merely some form of different ensemble representations of the same thing. These points are made carefully in $[5,18]$.

\subsection{Thermodynamic Information Geometry}

Motivated by Equation (10), we define the thermodynamic information metric elements as [20-22]

$$
g_{\alpha \beta}=-\frac{\partial^{2} S}{\partial X^{\alpha} \partial X^{\beta}} .
$$

This metric leads to the thermodynamic Ricci curvature scalar

$$
\begin{aligned}
& R=-\frac{1}{\sqrt{g}}\left[\frac{\partial}{\partial X^{1}}\left(\frac{g_{12}}{g_{11} \sqrt{g}} \frac{\partial g_{11}}{\partial X^{2}}-\frac{1}{\sqrt{g}} \frac{\partial g_{22}}{\partial X^{1}}\right)\right. \\
&\left.+\frac{\partial}{\partial X^{2}}\left(\frac{2}{\sqrt{g}} \frac{\partial g_{12}}{\partial X^{1}}-\frac{1}{\sqrt{g}} \frac{\partial g_{11}}{\partial X^{2}}-\frac{g_{12}}{g_{11} \sqrt{g}} \frac{\partial g_{11}}{\partial X^{1}}\right)\right]
\end{aligned}
$$

where $g=g_{11} g_{22}-g_{12}^{2}$. I use the sign convention of Weinberg [23], where $R$ for the two-sphere is negative. 
Numerous applications have demonstrated that $R$ gives information about the character of microscopic interaction forces; see [24] for a brief recent review. Near the critical point $|R|$ is proportional to $\xi^{d}$ :

$$
|R| \propto \xi^{d},
$$

with $\xi$ the correlation length, and $d$ the spatial dimensionality. The sign of $R$ is positive/negative for microscopic interactions repulsive/attractive. The situation is particularly clear in the case of pure fluids [25-27].

\subsection{Hyperscaling and the Geometric Equation}

A key ingredient from the theory of critical phenomena, one that completes the basic theory in this paper, is hyperscaling. Hyperscaling asserts that the singular part of the thermodynamic potential per volume $\phi$ is proportional to the inverse of the correlation volume $[4,28]$ :

$$
|\phi| \propto \frac{1}{\xi^{d}} .
$$

Combining this with Equation (15) leads to the geometric equation (GE):

$$
R=-\frac{\kappa}{\phi}
$$

where $\kappa$ is a dimensionless constant of order unity that the solution process always determines [29].

This loose derivation is presented only to give the reader some idea of the source of the GE Equation (17). In practical applications, it is the GE that is important. Its precise expression is shaped more by mathematical consistency in the solution process than by any derivation. If the reader wants, he or she may regard the GE as a postulate spanning a range of systems, whose validity is tested by the quality of its results. The precise definition of the free energy $\phi$ is taken from applications in ordinary thermodynamics:

$$
\phi=S-F_{1} X^{1}-F_{2} X^{2},
$$

where

$$
F_{\alpha}=\frac{\partial S}{\partial X^{\alpha}} .
$$

It has been argued that this GE extends in direct fashion to three or more independent variables [30]. For a detailed recent discussion of the expression and the justification of the GE, see $[31,32]$. These references also discuss the subtraction of a possible background term from $\phi$ (a subtraction not in play in this paper).

In ordinary thermodynamics, the metric elements and $\phi$ were always divided by the fixed volume, to make them scale invariant. However, a per volume representation is necessary neither for the calculation nor for the physical interpretation of $R$. In BHT, there is generally no constant scaling factor to divide the metric elements by, so I simply omit such a division. Without it, $R$ is dimensionless, and its physical interpretation is that its magnitude is proportional to the number of correlated Planck area pixels on the event horizon [33].

As is revealed by Equations (13), (14), (18) and (19), the GE may be written as a third-order partial differential equation (PDE) for $S$. [The fourth derivatives of $S$ subtract out in Equation (14).] Since both sides of Equation (17) are invariant scalar quantities, we could rewrite this equation in terms of other variables, yielding the same physical results. As I discuss below, I solve the GE only in cases where the entropy is assumed to be a generalized homogeneous function. This assumption simplifies the GE to a readily solvable third-order ordinary differential equation (ODE).

The GE has been solved in several ordinary thermodynamic scenarios, with good results; see Table 1 . Solutions were made in one or two analytic sections depending on whether or not 
there was a critical point at temperature other than zero. Expanding this list of solutions would further test the effectiveness of this method, and how reliable it might be in the black hole scenario.

Table 1. Tests of the GE in ordinary thermodynamics. $n$ denotes the number of independent thermodynamic variables, $d$ the spatial dimension, a\# the number of analytic sections, and $f$ the number of fit parameters for the scaled equation.

\begin{tabular}{lccccl}
\hline System & $n$ & $\boldsymbol{d}$ & $\boldsymbol{a \#}$ & $\boldsymbol{f}$ & Notes \\
\hline mean field theory [29] & 2 & - & 1 & 0 & exact \\
critical point [29] & 2 & 3 & 2 & 2 & $\chi^{2} \sim 1$ \\
galaxy clustering [34] & 2 & 3 & 1 & 0 & qualitative \\
corrections to scaling [30] & 3 & 3 & 2 & 3 & unclear \\
ideal gas paramagnet [35] & 3 & 3 & 1 & 0 & exact \\
power law interacting fluids [36] & 2 & 3 & 1 & 1 & qualitative \\
unitary fermi fluid [31,32] & 2 & 3 & 2 & 4 & $\chi^{2} \sim 2$ \\
1D ferromagnetic Ising [37] & 2 & 1 & 1 & 0 & exact \\
\hline
\end{tabular}

\subsection{Generalized Homogeneous Functions}

In this subsection, I discuss generalized homogeneous functions (GHF) of the form:

$$
\lambda S\left(X^{1}, X^{2}\right)=S\left(\lambda^{a_{1}} X^{1}, \lambda^{a_{2}} X^{2}\right),
$$

where $\lambda$ is a factor, and $a_{1}$ and $a_{2}$ are constant "critical exponents". This is equivalent to writing [38]

$$
S\left(X^{1}, X^{2}\right)=\left(X^{1}\right)^{a} Y\left[\frac{X^{2}}{\left(X^{1}\right)^{b}}\right]
$$

where $a=1 / a_{1}$ and $b=a_{2} / a_{1} . Y(z)$ is a function of a single variable:

$$
z=\frac{X^{2}}{\left(X^{1}\right)^{b}}
$$

It is easy to prove that if a function is a GHF, then both its derivatives are GHF's, and that multiplying, dividing, adding, or subtracting two GHF's results in a GHF. Hence, if $S\left(X^{1}, X^{2}\right)$ is a GHF, then the derived thermodynamic metric elements and the thermodynamic curvature are GHF's [18].

GHF's are at the foundation of the modern theory of critical phenomena [38]. They were also central to solving the GE for the systems in Table 1. GHF's also appear in the Kerr and the RN black holes discussed in the next subsection. Although I focus in this paper on the extremal black hole limit, non-extremal black hole phase transitions occur as well. These BHT phase transitions usually have the van der Waals mean field theory exponents, with the RN-AdS model serving as the prototype [39]. Potentially, the GE could describe all these phase transitions, and with critical exponents more general than those in mean field theory.

Åman et al. $[6,40]$ employed the scaled form in $d$ dimensions:

$$
\lambda^{d-2} S(M, J, Q)=S\left(\lambda^{d-3} M, \lambda^{d-2} J, \lambda^{d-3} Q\right),
$$

where $M$ is the mass, $J$ is the angular momentum, and $Q$ is the electric charge. $M$ is positive, but $J$ and $Q$ may have either sign. This scaling is satisfied by, for example, the Kerr-Newman black hole, an example that demonstrates nicely the unconventional BHT size scaling. However, the scaling in this paper is more general than that in Equation (23), and it is not tied to any scaling of the system size. 


\section{Canonical Classical Models}

In this section, I discuss two simple black hole solutions to the Einstein field equations: the Kerr and the RN black holes. I will refer to these solutions as the canonical classical models (CCM). Both models have GHF entropies.

The CCM, though not quantum, might offer some guidance about what to expect in real black holes. Their thermodynamic properties follow from the three variable Kerr-Newman black hole with $[41,42]$ :

$$
S(M, J, Q)=\frac{1}{8}\left(2 M^{2}-Q^{2}+2 \sqrt{M^{4}-J^{2}-M^{2} Q^{2}}\right) .
$$

I work in length units, with the conversion to real units described in, for example, Reference [18].

\subsection{Kerr Black Hole}

The Kerr black hole is perhaps the best model for real astrophysical black holes. It has $Q=0$, for which Equation (24) gives

$$
S(M, J)=\frac{1}{4} M^{2}\left(1+\sqrt{1-z^{2}}\right)
$$

where

$$
z=\frac{J}{M^{2}} .
$$

Clearly, the exponents in Equation (21) are $(a, b)=(2,2)$. Figure 3 shows six BHT functions for Kerr. These functions are all symmetric about $z=0$, with $-1<z<1$, and with $z= \pm 1$ corresponding to extremality $T=0$.
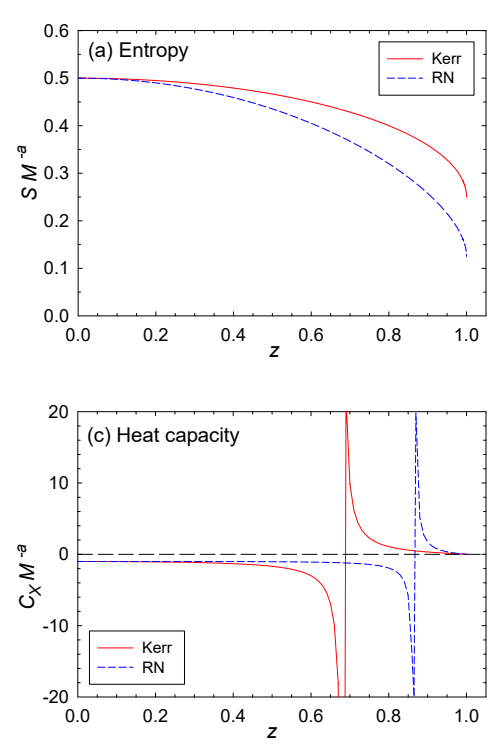
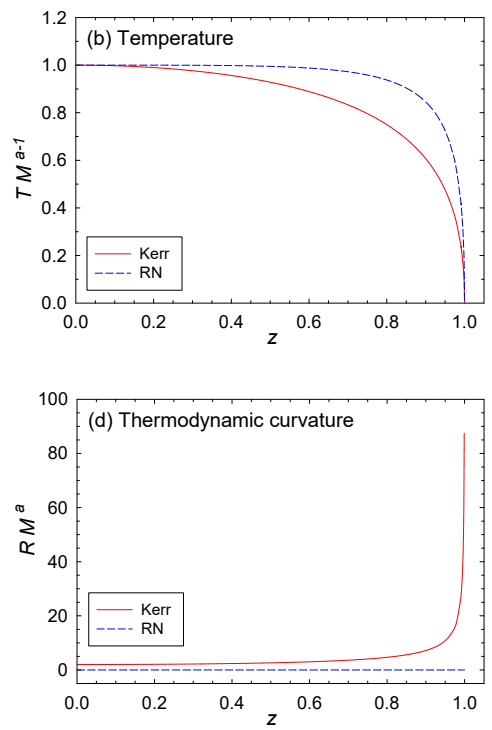

Figure 3. Cont. 

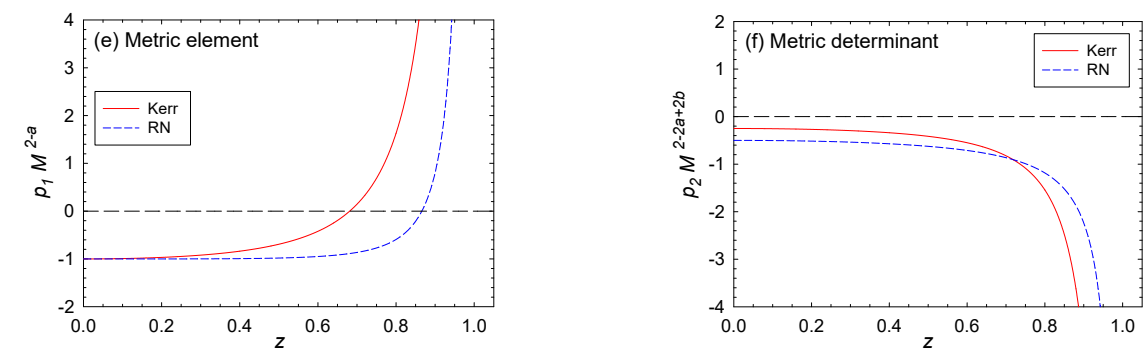

Figure 3. Six functions evaluated for Kerr and RN: (a) the entropy $S$; (b) the temperature $T$; (c) the heat capacity $C_{X}$, with $X=J$ or $Q ;(\mathbf{d})$ the thermodynamic curvature $R$; (e) the metric element $p_{1}$; and (f) the metric determinant $p_{2}$. With the exception of $R$, Kerr and RN show qualitatively similar behavior.

\subsection{Reissner-Nordström Black Hole}

The RN black hole has $J=0$, for which Equation (24) gives

$$
S(M, Q)=\frac{1}{8} M^{2}\left(2-z^{2}+2 \sqrt{1-z^{2}}\right),
$$

where

$$
z=\frac{Q}{M}
$$

Clearly, the exponents in Equation $(21)$ are $(a, b)=(2,1)$. Figure 3 shows six functions for RN. As with Kerr, these functions are symmetric about $z=0$, with $-1<z<1$, and with $z= \pm 1$ corresponding to extremality. For $R N$, we have directly from Equation (14) that $R=0$. Indeed, Åman et al. [40] placed scaled forms of the entropy with $b=1$ into a special category having flat geometries.

\subsection{Some General Observations}

The classical CCM lack quantum properties, and so their physical validity is logically open to question. Quantum properties at the microscopic level do not necessarily disappear in the limit of many degrees of freedom. For example, there are cases in ordinary thermodynamics where quantum properties persist even at large scales; for example, superconductivity and superfluidity. However, in the context of the AdS/CFT correspondence, classical black holes do describe a well-defined limit of the underlying quantum theory.

These issues aside, we nevertheless hope that the CCM offer at least some guidance for understanding real scenarios. Since the thermodynamic approach described in this paper offers many possible solutions, most of them probably not physical, the choices must be narrowed. My try is to generate solutions over a grid of reasonable $(a, b)$ values, and then to focus on the solutions that approximate most closely our expected black hole properties. The hope is that the true black hole behavior will be among these solutions. My discussion is limited to two independent variables, and to certain assumptions about the symmetry and analyticity of the solutions, but other choices are certainly possible.

Experimental black hole data would be of great value in narrowing down the choices of possible solutions. Data is emerging from measurements of gravitational waves [43] and from tidally distorted events [44]. In addition, there are a host of good string theory models on the scene. The structure outlined in this paper could form a context for analyzing information from various sources.

Here are several general observations about the CCM. Henceforth, I use the notation $M=X_{1}$ and $X=X_{2}$.

1. $S$ is an even GHF of $z=X M^{-b}$, and analytic at $z=0$. See Equations (25) and (27). 
2. The CCM have sharp extremal edges. See Figure 3a,b. As $z$ gets larger, $S M^{-a}$ decreases from maxima at $z=0$ to finite positive values at $z=1$ at which $T \rightarrow 0$. This point marks the extremal point, where the surface gravity is expected to be zero [1].

3. As $M \rightarrow 0, T \rightarrow \infty$ only if $a>1$. See Equation (A5). This limit is Hawking's well-known result.

4. The heat capacities $C_{X}$ start negative. See Figure $3 \mathrm{c}$. This negative start reflects the well-known lack of thermodynamic stability of spherical self-gravitating objects.

5. The heat capacities $C_{X}$ have infinities unconnected with the extremal limit at a single value of $|z|<1$. See Figure 3c. These infinities correspond to a change in sign of $C_{X}$ from negative to positive as $z$ increases. The heat capacities go to zero in the extremal limit $|z|=1$.

6. The Kerr extremal black hole has thermodynamic curvature $R \rightarrow+\infty$. See Figure 3d. In ordinary thermodynamics, the Bose/Fermi ideal quantum gases have $R$ diverging to minus/plus infinity as $T \rightarrow 0$, and the effects of the quantum statistical interactions become more pronounced [45,46]. $R \rightarrow \infty$ as $T \rightarrow 0$ indicates that the conjectured fundamental particles constituting Kerr might be fermionic [33].

7. The $R N$ black hole has $R=0$. See Figure $3 d$. This result suggests a classical non-quantum ideal gas as constituting the microscopic constituents. Such an idea may strike one as demonstrably unphysical [47] since we associate black holes with strong gravitational interactions. However, the gravitational component might be non-statistical, associated perhaps with the massive, central singularity predicted by general relativity.

8. There are no regimes of thermodynamic stability. See Figure 3e,f. In particular, $p_{2}$ is always negative.

\subsection{Additional Comments}

The sign change, and the corresponding plus and minus infinities, in the heat capacity $C_{X}$ have been interpreted as indicating phase transitions by Davies [41]. However this interpretation has been questioned, since this sign change does not correspond to a change in thermodynamic stability [19]. Nevertheless, the sign change in $C_{X}$ is a persistent feature in BHT, and I will come back to it below.

There are certainly BHT models with true, non-extremal, phase transitions. Examples are the RN-AdS black hole, with a van der Waals type phase transition, and matter-free AdS space with a Hawking-Page phase transition between black hole/no black hole solutions. Results in the literature have $R$ typically negative at the der Waals type BHT phase transitions [19,48-50], the same sign as the critical phenomena models in ordinary thermodynamics. The Hawking-Page phase transition has been associated with $R=0$ [49].

Phase transitions other than extremal do not appear in my solutions below. However, the method laid out here could be readily extended to include such cases.

\section{Calculation Method}

In this section, I solve the GE in the context of the scaling relation Equation (21) that in the present notation is

$$
S=M^{a} Y\left(\frac{X}{M^{b}}\right) .
$$

Equations (13) and (14) yield the thermodynamic curvature $R$, and Equations (18) and (19) yield the free energy $\phi$. Since $R$ has derivatives of $S$ up to third order in $Y(z)$, the resulting GE Equation (17) becomes a third-order ODE for $Y(z)$.

Motivated by observation 1 in Section 3.3, I assume that $Y(z)$ is an analytic, even function at $z=0$, with series

$$
Y(z)=y_{0}+y_{2} z^{2}+y_{4} z^{4}+\cdots,
$$

where $y_{0}, y_{2}, y_{4}, \ldots$ are the constant series coefficients. Substituting this series into $R$ and $\phi$ yields

$$
R M^{a}=-\frac{(b-1)(a-2 b)}{(a-1) a y_{0}}+\frac{\mathcal{N}_{2}}{(a-1)^{2} a^{2} y_{0}^{2} y_{2}} z^{2}+O\left(z^{4}\right),
$$


where

$$
\begin{aligned}
\mathcal{N}_{2}=2(b-1) \times & \\
& \left\{-a^{3}\left(y_{2}^{2}-3 y_{0} y_{4}\right)+a^{2}\left([6 b-1] y_{2}^{2}-3 y_{0} y_{4}\right)+4 a(1-3 b) b y_{2}^{2}+4 b^{2}(2 b-1) y_{2}^{2}\right\},
\end{aligned}
$$

and

$$
\frac{M^{a}}{\phi}=-\frac{1}{(a-1) y_{0}}+\frac{y_{2}(1+a-2 b)}{(a-1)^{2} y_{0}^{2}} z^{2}+O\left(z^{4}\right)
$$

Substituting these two series into the GE Equation (17), and equating the zero'th order terms, yields

$$
\kappa=-\frac{(b-1)(a-2 b)}{a}
$$

independent of any series coefficients. Since the GE is a third-order ODE, three free constants are required for its integration. One of these constants has already been set to zero to make the series even. Specifying $y_{0}$ and $y_{2}$ now leads to a complete series solution, since equating the second-order series terms yields $y_{4}$, and equating higher-order terms yields all of the remaining coefficients. This series solution may be used to calculate a quadruple of values $\left\{z_{0}, Y\left(z_{0}\right), Y^{\prime}\left(z_{0}\right), Y^{\prime \prime}\left(z_{0}\right)\right\}$ that serve as the initial conditions for a numerical solution of the GE for any values of $\left\{a, b, y_{0}, y_{2}\right\}$.

Generally, we may easily prove that for any $(a, b)$, and for any nontrivial pair of constants $n_{1}$ and $n_{2}$, if $Y(z)$ is a solution, then $n_{1} Y\left(n_{2} z\right)$ is a solution [30]. In critical phenomena theory such a result is called universality since it allows for several different solutions with the same critical exponents and the same function $Y(z)$. Whether or not this universality has physical significance in the BHT scenario is not yet clear. However, it simplifies the solutions process, since to find scaled solutions $Y(z)$ it suffices merely to give values for $(a, b)$ and to specify the signs of $y_{0}$ and $y_{2}$.

I conclude this section by writing the full ODE for the exponent values $(a, b)=(2,2)$ :

$$
Y^{(3)}(z)=\frac{z Y^{\prime \prime}(z)^{2}}{z Y^{\prime}(z)-Y(z)}
$$

To write the ODE for general $(a, b)$ takes too much space (The denominator of Equation (35) is proportional to the inverse temperature given in Equation (A5) for $(a, b)=(2,2)$. However, this proportionality does not generalize to other values of $(a, b)$, so it is of little interest.).

\section{Results}

In this section, I discuss results. I confined my search of exponent values to the rectangle with diagonal corners at $\{a, b\}=\{1,1\}$ and $\{3,5 / 2\}$. The lower exponent limits come from the considerations discussed below, and the upper limits were set to avoid drifting too far from the CCM exponents.

\subsection{General Comments}

The GE admits a broad scope of possible solutions, reflecting the multiple physical possibilities for microscopic forces. However, in the presence of strong effective microscopic forces (i.e., those producing long-range correlations), the system arranges itself into spatially large mesoscopic structures of size $\xi$, in which the details of the microscopic forces are not important. This is the basic justification in the modern theory of critical phenomena for regarding only the dimensionalities of the physical space and of the order parameter to be relevant for determining the universal properties [38]. The result is a great simplification in the form of scaled universal expressions for the free energy. The information geometry of thermodynamic adds to this picture an explicit method of generating the scaled free energy in terms of the critical exponents. 
Since scaled expressions for the thermodynamic properties are also frequent occurrences in BHT (see Section 3), we might infer that the unknown underlying microscopic black hole constituents also interact strongly. In this event, the basic philosophy of critical phenomena holds for BHT, and the program for generating scaled solutions offered by solving the GE might prove effective. The expression in Equation (29), inspired by the CCM, seems the simplest try for a scaled solution.

As I argued in Section 4, the only free parameters in the scaled solutions of the GE Equation (17), other than the simple scaling factors $n_{1}$ and $n_{2}$ in Section 4 (simply related to $y_{0}$ and $y_{2}$ ), are $(a, b)$, and the signs of $y_{0}$ and $y_{2}$. Guided by the considerations of Section 3 , I will attempt a narrowing of the acceptable values of $(a, b)$.

\subsection{The Signs of $y_{0}$ and $y_{2}$}

Start by considering the signs of $y_{0}$ and $y_{2}$. First, notice from the series for $T$ Equation (A6) in the Appendix that Hawking's result $T \rightarrow \infty$ as $M \rightarrow 0$ requires $a>1$. Include the series for $S$ Equation (A4), and we see that positive $S$ and $T$ at $z=0$ requires positive $y_{0}$. Positive $y_{0}$ and $a>1$ are thus required. From the series Equation (A10), $C_{X}$ will now be negative at $z=0$, a feature also of the CCM.

The sign of $y_{2}$ is equally clear. The series for $S$ has $S M^{-a}$ be a local maximum at $z=0$ if and only if $y_{2}$ is negative. Such local maximas in $S$ obtain in the CCM. Furthermore, detailed numerical solutions to the GE for the $(a, b)$ values considered here show that $S$ is monotonically decreasing with increasing $z$ if and only if $y_{2}$ is negative. I thus consider only negative $y_{2}$.

For given $(a, b), Y(z)$ for any $\left(y_{0}, y_{2}\right)$ may be scaled to $Y(z)$ for any other $\left(y_{0}, y_{2}\right)$ with the same signs using the scaling relation Equation (20). Therefore, with no loss of generality, I take $y_{0}=1$ and $y_{2}=-1$ in all of my solutions below.

\subsection{The Solution Types}

The scaled solutions to the GE fall into basic types according to the values of $(a, b)$. I list these types in this subsection. Types $4-6$ seem the most physically relevant. Figure 4 sketches the types, and the corresponding regimes of $(a, b)$ space where they obtain.
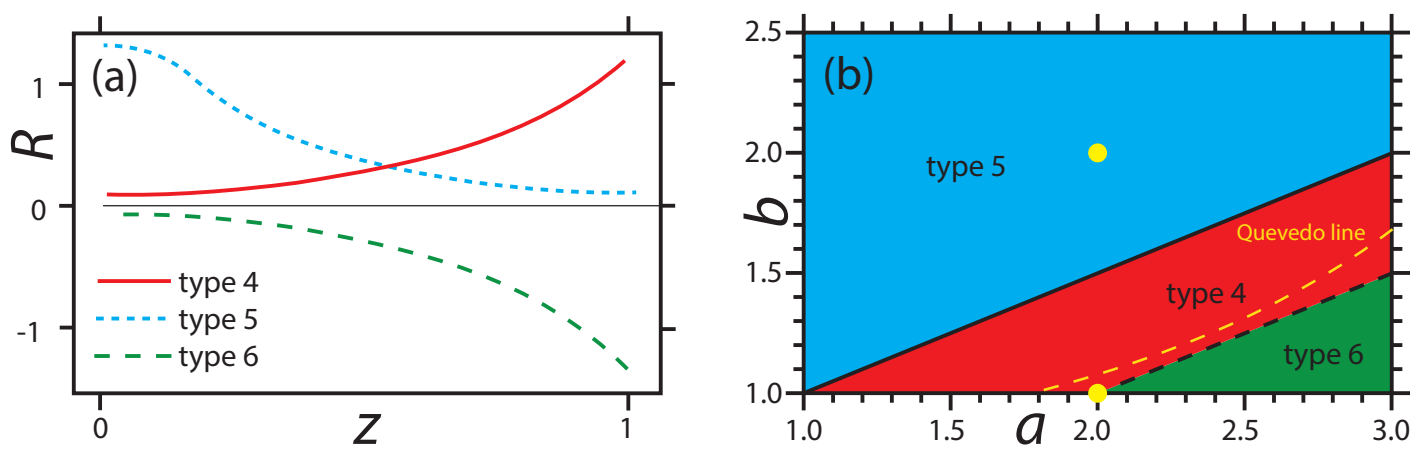

Figure 4. Types of functions for $R$ sketched as $z$ increases from zero: (a) type 4 (red) has $R$ diverging to $+\infty$ at some finite $z$; type 5 (blue) has $R$ going asymptotically to zero; and type 6 (green) has $R$ diverging to $-\infty$ at some finite $z$. (b) The regimes in $(a, b)$ space for which each type obtains. Along the solid line, $R$ is constant but not zero (type 3), and along the dotted line, $R=0$ (type 2). The axis $b=1$ is type 1 . The bold yellow dots correspond to the $(a, b)$ values of $\operatorname{Kerr}(2,2)$ and $R N(2,1)$. The dashed yellow curve is the Quevedo line where $C_{X}$ and $R$ diverge at the same $z$. Type 4 seems physically the most relevant.

Type 1 has $b=1$, corresponding to $\kappa=0$, by Equation (34). In this case, the GE clearly has $R=0$. Furthermore, $R=0$ no matter what the function $Y(z)$. The case $b=1$ is a special category identified 
by Åman et al. [40] as having flat geometry. Since the GE tells us nothing about $Y(z)$ for $b=1$, I will not consider type 1 further.

Type 2 has $a=2 b$, also corresponding to $\kappa=0$, and thus to $R=0$. One can show, however, that type 2 does not allow just any $Y(z)$, and that the even solution to $R=0$ is

$$
Y(z)=y_{0}+y_{2} z^{2}
$$

with $y_{0}$ and $y_{2}$ free constants. This solution yields

$$
T=\frac{M^{1-2 b}}{2 b y_{0}},
$$

independent of $z$. A $T$ independent of $z$ is quite unlike the $T^{\prime} s$ of the CCM in Figure 3b. Type 2 is then perhaps not very physically relevant.

Type 2a has $(a, b)=(2,1)$ (the RN exponents), and so it is both type 1 and type 2 . However, any function $Y(z)$ has $R=0$ for all three cases: $(a, b)=(2,1),(a, b)=\operatorname{Limit}[(a, 1) \rightarrow(2,1)]$, and $(a, b)=\operatorname{Limit}[(2, b) \rightarrow(2,1)]$. Hence, this hybrid case actually corresponds to type 1 .

Type 3 has $a=2 b-1$. The simple quadratic expression for $Y(z)$ in Equation (36) yields

$$
R=\frac{M^{1-2 b}}{2(2 b-1) y_{0}},
$$

a constant independent of $z$. It also yields

$$
\phi=-2 M^{2 b-1}(b-1) y_{0} .
$$

Type 3 thus has even solution $Y(z)=y_{0}+y_{2} z^{2}$, with $\kappa=(b-1) /(2 b-1)$, a value for $\kappa$ consistent with Equation (34). Furthermore,

$$
S=M^{2 b-1}\left(y_{0}+y_{2} z^{2}\right),
$$

and

$$
T=\frac{M^{2(1-b)}}{(2 b-1) y_{0}-y_{2} z^{2}} .
$$

With $y_{0}>0$ and $y_{2}<0$, the expressions for $S$ and $T$ have features in common with the CCM, and thus type 3 might have some physical interest.

Turn now to the three other distinctive types 4-6. For guidance, refer to the ODE in Equation (35), given for the case with $(a, b)=(2,2)$. Key here, and for more general values of $(a, b)$, is whether or not the denominator on the right-hand side crosses through zero. Unless the numerator is also zero at this crossing point, the result is a diverging $Y^{(3)}(z)$. Since $Y^{(3)}(z)$ is a factor in the numerator of $R$, but not in its denominator [see Equation (A15)], the zero crossing has $R$ also diverging. I identify three types of divergences: $R$ diverging positive (type 4 ), negative (type 6), or $R$ not diverging at all (type 5 ). I denote by $z=z_{R}$ the position of a possible divergence of $R$.

A diverging $R$ signals a nonanalytic solution for $Y(z)$. A number of examples with nonanalytic solutions were found in the ordinary thermodynamic examples shown in Table 1 . There, they corresponded to phase transitions, and in some cases bridging techniques for continuing solutions beyond these phase transitions were found. However, there is too little guidance from the CCM to attempt such a bridging here, and I simply terminate solutions at points of diverging $R$. On the first try, it seems natural to associate these divergences with the extremal limit.

Type 4 has $(a+1)>2 b>a$. $R$ starts positive at $z=0$, and then increases monotonically to $+\infty$ at some value $z=z_{R}$. This case matches qualitatively the situation in Kerr in Figure 3d, so type 4 might be a serious candidate for real black holes. 
Type 5 has $2 b>(a+1) . R$ starts positive at $z=0$, and then goes asymptotically to zero with increasing $z$. Physically, $R$ going to zero asymptotically speaks to interparticle forces diminishing in the extremal limit. For type 5 , the solutions stay regular all the way out to $z \rightarrow \infty$. However, we expect big spin and/or big charge to lead to a black hole breakup at some finite $z$. Hence, type 5 might not be physically plausible.

Type 6 has $2 b<a$. $R$ starts negative at $z=0$, and then decreases monotonically to $-\infty$ at some value $z=z_{R}$. Type 6 does not match the CCM, so it is harder to fit it into a possible physical picture. Negatively diverging $R$ 's speak to critical phenomenon type phase transitions, with effectively long-range attractive forces between microscopic constituents producing long-range order. Such phase transitions certainly take place in BHT models [39,49], but they are typically associated with more independent thermodynamic variables or with AdS backgrounds, neither present in this paper. Type 6 also has $T$ increasing monotonically with $z$, quite unlike the decrease seen in the CCM. Hence, I do not regard type 6 as physically plausible.

Figure 5e shows $R$ for $(a, b)=(1.9,1.4)$ (type 4$)$. Figure $5 f$ shows the values $z=z_{R}$ where $|R|$ diverges for types 4 and 6 . I consider the regime with $z>z_{R}$ to be unphysical because $Y(z)$ ceases to be analytic at $z=z_{R}$. Again, type 5 has $z_{R} \rightarrow \infty$.

(a)

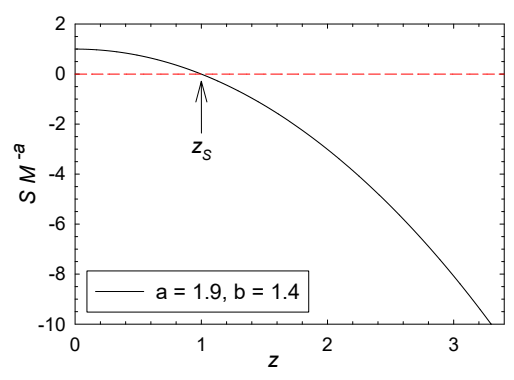

(c)

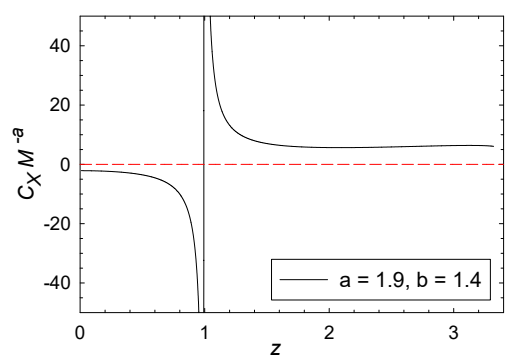

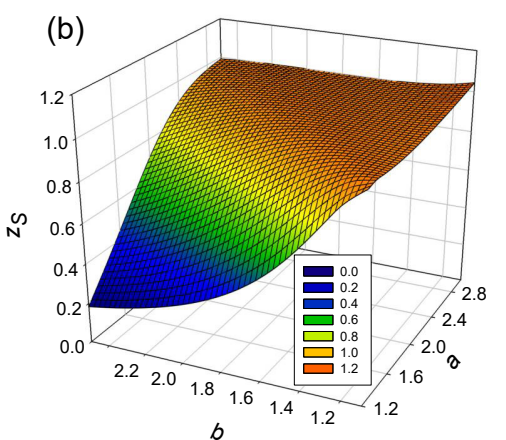

(d)

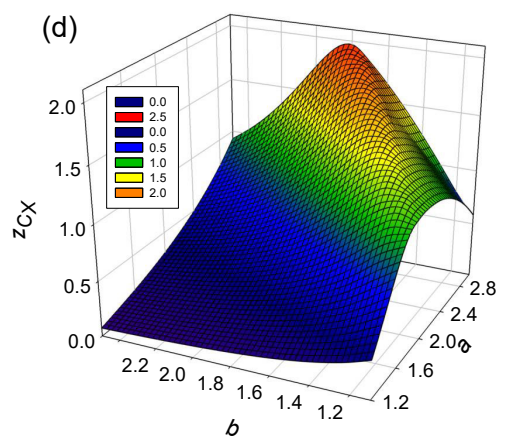

Figure 5. Cont. 
(e)

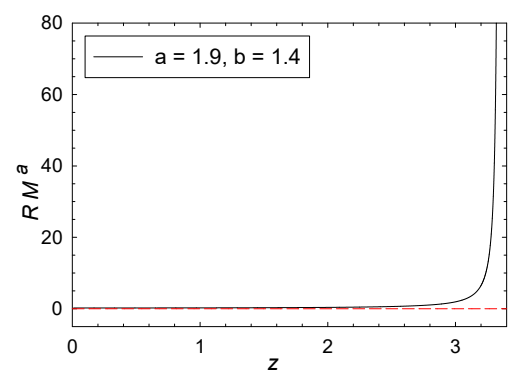

(f)

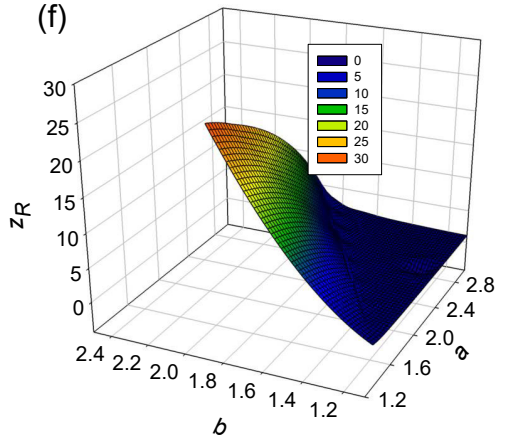

Figure 5. Axis crossing $z_{S}$ and the divergence positions $z_{C_{X}}$ and $z_{R}$. Also shown are representative cases with $a=1.9$ and $b=1.4$, having $z_{C_{X}}=0.9970$ and $z_{R}=3.326$. (a,b) represent the entropy $S$, with the $z$ axis crossings at $z=z_{S} ;(\mathbf{c}, \mathbf{d})$ represent the heat capacity $C_{X}$, with the divergence positions at $z=z_{C_{X}}$ for the $(a, b)$ values corresponding to $z_{C_{X}}<z_{R}$; and (e,f) represent the thermodynamic curvature $R$, with the divergence positions at $z=z_{R}$ for types 4 and 6 . For type $5, z_{R} \rightarrow \infty$.

\subsection{Further Discussion of Solution Types}

The simplest thermodynamic variable is $S$. For all of the values of $(a, b)$ considered here, $S=M^{a} Y(z)$ shows the qualitative behavior in Figure 5a. For small $z$, the series Equation (A4) has $Y(0)=1$, with $Y(z)$ decreasing monotonically with increasing $z$. Numerical solutions continue to show this monotonic decrease, with $Y(z)$ crossing the $z$ axis at some point $z=z_{S}$. Figure $5 \mathrm{~b}$ shows $z_{\mathcal{S}}$ as a function of $(a, b)$. All cases have $z_{S}<z_{R}$.

The value $S=0$ could be taken to mark the absolute lower limit of the value of $S$, since negative entropies are hard to interpret physically. However, negative BHT entropies have been found as well in charged Gauss-Bonnet dS/AdS gravity by Odintsov et al. [51,52]. These negative entropies were interpreted as marking either a regime where parameter values have transitioned into an unacceptable range, or into a regime where there is some sort of a phase transition. Clunan et al. [53] argued that negative entropies in Gauss-Bonnet gravity could be eliminated by adding to $S$ a constant positive factor. Such an addition certainly fits the spirit of the theory of critical phenomena in which only the "singular parts" of quantities are considered.

The scaled behavior of $S$ in Figure 5a shows some qualitative features in common with those of the CCM in Figure 3a. However, $S$ for the CCM terminates at a value for $z$ with infinite slope, $Y^{\prime}(z) \rightarrow-\infty$. This infinite slope leads to $T=0$, by Equation (A5). $T=0$ corresponds to the extremal limit in the CCM, with $T$ proportional to the surface gravity.

The extremal limit from the solutions to the GE is less well defined. The character of $T$ is indicated by the series Equation (A6). Clearly, with $z$ increasing from $z=0, T$ decreases if $2 b>a$ (corresponding to types 4 and 5 in Figure 4), and increases if $2 b<a$ (corresponding to type 6). The full solution to the GE continues this monotonic decrease to larger $z$, including constant $T$ for $2 b=a$. Clearly, type 6 with its monotonically increasing $T$ is physically unsatisfactory.

The final element of my picture is the divergence of the heat capacity $C_{X}$, with the case for the CCM shown in Figure 3c. Many GE solutions on my $(a, b)$ grid show such a divergence; see Figure $5 c$ for a representative example. Neither for the CCM nor for the GE solutions does the divergence of $C_{X}$ typically correspond either to a divergence of $R$ or to a breakdown of analyticity in $Y(z)$. $C_{X}$ diverges only because its denominator passes through zero and changes sign. Nevertheless, the BHT literature pays considerable attention to the divergence of $C_{X}$, so I will do likewise.

An interesting question is whether the divergence of $C_{X}$ at $z=z_{C_{X}}$ happens prior to that of $R$ at $z=z_{R}$. Both types 4 and 6 have finite $z_{R}$, and for the infinity in $C_{X}$ to be in the physical regime, as it is for the CCM, we must have $z_{C_{X}}<z_{R}$. Figure $5 d$ shows $z_{C_{X}}$ as a function of $(a, b)$ for cases with $z_{C_{X}}<z_{R}$. Type 5 is analytic all the way out to infinity, so for type 5 we could have $z_{C_{X}} \rightarrow \infty$. 
The limiting curve in the $(a, b)$ plane with $z_{C_{X}}=z_{R}$ may be of interest to researchers who argue that a true BHT phase transition should have $C_{X}$ and $R$ diverging in the same place; see Quevedo [47]. If this overlap of divergences is physically necessary, then the curve of overlap would logically correspond to the locus of true physical black holes. This curve is shown in Figure 4, with points above the curve corresponding to $z_{C_{X}}<z_{R}$.

Figure 6 plots $T$ and $R$ for $b=1.3$ and several values of $a$. Solutions for $Y(z)$ become nonanalytic where $|R|$ diverges. I did not extend my solutions beyond these points of non-analyticity, since there is too little guidance from the CCM for doing so. Types 3 and 5 have no diverging $R$ values, and value of $T$ and $R$ rapidly approach zero as $z \rightarrow \infty$.
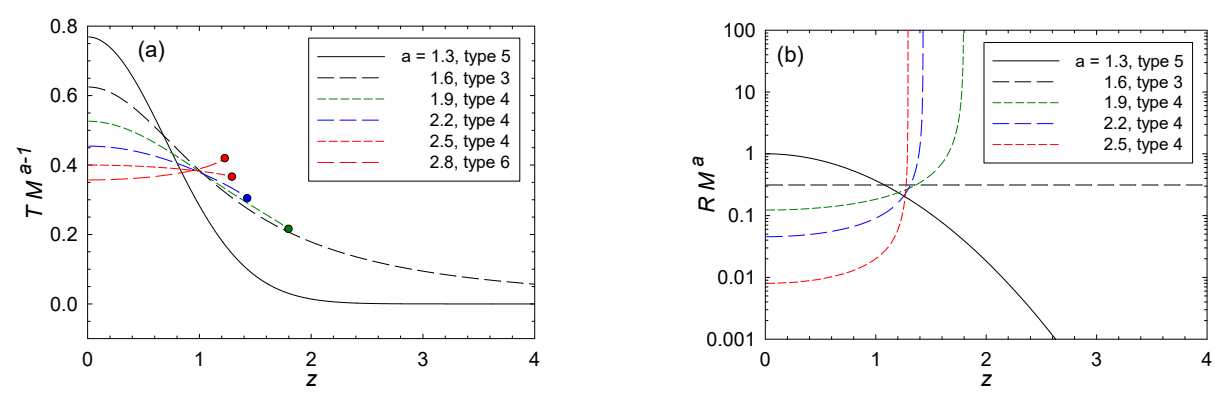

Figure 6. Graphs for $T$ and $R$ with $b=1.3$ and increasing values of $a$ : (a) shows $T$ as a function of $z$ and (b) shows $R$ as a function of $z$, with each function scaled by an appropriate factor of $M$ to make them dimensionless. The graphs show instances of types $3,4,5$, and 6 . Solutions for $Y(z)$ cease to be analytic when $R$ diverges to either $+\infty$ or $-\infty$ (the type 6 divergence of $R$ is not shown on the log scale since $R$ is negative). These infinities in $|R|$ mark points of termination, indicated on the $T$ graphs by bold dots. Type 3 and 5 curves do not terminate.

\subsection{Final Remarks about the GE Solutions}

The GE solutions have produced candidate BHT equations of state in qualitative agreement with several features of the CCM. All of the GE solutions in my search grid have the entropy $S$ decreasing monotonically from maxima at $z=0$. With appropriate values of $(a, b)$, there are several GE solutions of types 4-6 with $C_{X}$ starting negative, diverging to negative infinity, and then returning from positive infinity as $z$ increases. GE solutions of all types except type 1 have metric determinants $p_{2}$ uniformly negative. GE solutions of types 4 and 5 have $T$ decreasing monotonically from maxima at $z=0$. Type 4 has positive values of $R$ diverging to infinity, matching qualitatively a basic feature of Kerr in the $\mathrm{CCM}$. These are all encouraging results, particularly considering that just the two exponents $(a, b)$ were varied.

Areas of difference concern the character of the BHT extremal point. In the CCM, general relativity has $T \rightarrow 0$ correspond to zero surface gravity, and, at least for Kerr, $R \rightarrow \infty$. As was pointed out by Ruppeiner [33], this extremal behavior for Kerr has features in common with the ideal Fermi gas. However, the CCM entropies do not go to zero in the extremal limit, inconsistent with usual statements [3] of the third law of thermodynamics that has $S \rightarrow 0$ as $T \rightarrow 0$. This discrepancy results from the event horizon area (proportional to $S$ ) not going to zero in this limit.

The GE solutions show a somewhat different extremal limit. $S$ decreases monotonically to zero, but the zero coincides neither with any zero in $T$ nor to a divergence in $R$. Although the position of this entropy zero might be adjustable, the CCM gives no particular insight about how this should be done. Therefore, I will not pursue this further here. Generally, types 4 and 5 seem physically the most interesting. 


\section{Model Comparisons}

The main agenda of this paper is to get the BHT equations of state by thermodynamic means. However, there is another method of proceeding: BHT solutions derived from known black hole models can be tested for agreement with the GE. A detailed exploration of this theme is beyond the scope of this paper, but this section presents a brief review of the large recent literature of BHT papers computing the thermodynamic curvature $R$ from various classical and quantum models. An example of comparing model solutions with the GE was by Sahay and Jha [54] who demonstrated that near the phase transition in the $5 \mathrm{~d}$ Gauss-Bonnet-AdS black hole, the free energy expression satisfies the GE.

In the general relativistic scenario, most of the recent models have added AdS backgrounds. AdS backgrounds bring thermodynamic stability, Hawking-Page phase transitions, and connections to AdS/CFT correspondence. These classical scenarios have seen extensions of spatial dimensionality via the Myers-Perry black holes [55,56], and via exotic local topologies [57,58]. The issue of the appropriate number of independent variables to use has been raised [59]. Classical RN-AdS black holes have van der Waals phase transitions $[39,48-50,60]$. Attention has been paid to adding the pressure as a thermodynamic variable, with the conjugate variable being the volume enclosed by the black hole [61,62].

Also on the scene is a broad class of models appending terms quadratic and higher-order in the space-time Ricci curvature scalar to the Einstein-Hilbert Lagrangian. These approaches usually also employ Maxwell terms, and contain free parameters calculable by string theory. Such models include Gauss-Bonnet AdS gravity [54,63], Hořava-Lifshitz gravity [64,65], $f(R)$ gravity [66,67], and Einstein-dilaton-Lifshitz gravity [68]. Another class of black holes to which geometry of thermodynamics has been applied employs nonlinear electrodynamics (NED) [69]. There have also been efforts to model BHT phase transitions with Ehrenfest's theory of phase transitions [70,71]. In addition, some authors have used different thermodynamic metrics in the black hole regime [47,72].

\section{Conclusions}

In this paper, I have argued that fundamental properties about black hole thermodynamics might be obtained by thermodynamic means. General assumptions about the number of conserved thermodynamic variables, conditions of analyticity, and limiting cases, yield a scaled thermodynamics as a solution to an ordinary differential equation parameterized by two exponents $(a, b)$. The basic agenda proposed here is that information about black holes from either models or from astrophysical observations could serve to constrain $(a, b)$ and correspondingly improve our knowledge of black hole thermodynamics in real physical scenarios.

Because the true black hole thermodynamic equations of state are so hard to get, in the absence of a consensus microscopic model, the method here is hard to test directly. However, the same method may be employed in ordinary thermodynamics, where there is a good grounding on statistical mechanics. Several successful tests of my method have been made in this conventional scenario. If we believe in the unity of thermodynamics, there may be reason to think that the method proposed here will be effective in a broader scenario, including black holes.

Acknowledgments: I thank Anurag Sahay for useful conversations at the SigmaPhi 2017 conference, and Alex Roman for locating several references.

Conflicts of Interest: The author declares no conflict of interest.

\section{Appendix A. General Formulas}

In this section, I list formulas and series for several quantities. The series are generated starting with

$$
Y(z)=y_{0}+y_{2} z^{2}+y_{4} z^{4}+O\left(z^{6}\right)
$$


where

$$
z=\frac{X}{M^{b}} .
$$

In this paper, I consider without significant loss of generality only $y_{0}=1$ and $y_{2}=-1$. The entropy is

$$
S M^{-a}=Y(z),
$$

with

$$
S M^{-a}=y_{0}+y_{2} z^{2}+O\left(z^{4}\right)
$$

The temperature is

$$
T M^{a-1}=\frac{1}{a Y(z)-b z Y^{\prime}(z)}
$$

with

$$
T M^{a-1}=\frac{1}{a y_{0}}-\frac{y_{2}(a-2 b)}{a^{2} y_{0}^{2}} z^{2}+O\left(z^{4}\right) .
$$

The free energy is

$$
\phi M^{-a}=\left[(b-1) z Y^{\prime}(z)-(a-1) Y(z)\right]
$$

with

$$
\phi M^{-a}=-(a-1) y_{0}-(1+a-2 b) y_{2} z^{2}+O\left(z^{4}\right) .
$$

The heat capacity at constant $X$ is

$$
C_{X} M^{-a}=-\frac{\left[a Y(z)-b z Y^{\prime}(z)\right]^{2}}{b z\left[(-2 a+b+1) Y^{\prime}(z)+b z Y^{\prime \prime}(z)\right]+(a-1) a Y(z)},
$$

with

$$
C_{X} M^{-a}=-\frac{a y_{0}}{a-1}-\frac{y_{2}\left[a^{2}-a+2(1-2 b) b\right]}{(a-1)^{2}} z^{2}+O\left(z^{4}\right) .
$$

The first determinant coefficient is

$$
p_{1} M^{2-a}=a(1-a) Y(z)-b z\left[(1-2 a+b) Y^{\prime}(z)+b z Y^{\prime \prime}(z)\right],
$$

with

$$
p_{1} M^{2-a}=-a(a-1) y_{0}+y_{2}\left(-a^{2}+4 a b+a-4 b^{2}-2 b\right) z^{2}+O\left(z^{4}\right) .
$$

The second determinant coefficient is

$$
p_{2} M^{2-2 a+2 b}=-(a-b)^{2} Y^{\prime}(z)^{2}+a(a-1) Y(z) Y^{\prime \prime}(z)-b(b-1) z Y^{\prime}(z) Y^{\prime \prime}(z),
$$

with

$$
p_{2} M^{2-2 a+2 b}=2 a(a-1) y_{0} y_{2}-2\left(a^{2} y_{2}^{2}-4 a b y_{2}^{2}+a y_{2}^{2}+4 b^{2} y_{2}^{2}-2 b y_{2}^{2}+6 a y_{0} y_{4}-6 a^{2} y_{0} y_{4}\right) z^{2}+O\left(z^{4}\right) .
$$

The thermodynamic curvature is

$$
R M^{a}=\frac{\mathcal{N}_{1}}{\mathcal{D}_{1}},
$$

where 


$$
\begin{aligned}
& \mathcal{N}_{1}=-(b-1) \times \\
& \left\{-Y^{\prime}(z)\left[b z\left(a^{2}-3 a b+2 b\right) Y^{\prime \prime}(z)^{2}+(a-1) a(a-b) Y(z) Y^{(3)}(z)-(b-1) b^{2} z^{2} Y^{(3)}(z) Y^{\prime \prime}(z)\right]+\right. \\
& (b-a) Y^{\prime}(z)^{2}\left[\left(a^{2}-2 a b-a+b^{2}+b\right) Y^{\prime \prime}(z)+b z(-2 a+b+1) Y^{(3)}(z)\right]+ \\
& \left.(a-1) a Y(z) Y^{\prime \prime}(z)\left[2(a-2 b) Y^{\prime \prime}(z)-b z Y^{(3)}(z)\right]-(b-1) b^{2} z^{2} Y^{\prime \prime}(z)^{3}\right\},
\end{aligned}
$$

and

$$
\mathcal{D}_{1}=2\left[(a-b)^{2} Y^{\prime}(z)^{2}-(a-1) a Y(z) Y^{\prime \prime}(z)+(b-1) b z Y^{\prime}(z) Y^{\prime \prime}(z)\right]^{2} .
$$

The series for $R$ is given in Equation (31):

$$
R M^{a}=-\frac{(b-1)(a-2 b)}{(a-1) a y_{0}}+\frac{\mathcal{N}_{2}}{(a-1)^{2} a^{2} y_{0}^{2} y_{2}} z^{2}+O\left(z^{4}\right),
$$

with

$$
\begin{aligned}
\mathcal{N}_{2}=2(b-1) \times & \\
& \left\{-a^{3}\left(y_{2}^{2}-3 y_{0} y_{4}\right)+a^{2}\left[(6 b-1) y_{2}^{2}-3 y_{0} y_{4}\right]+4 a(1-3 b) b y_{2}^{2}+4 b^{2}(2 b-1) y_{2}^{2}\right\} .
\end{aligned}
$$

\section{References}

1. Wald, R.M. The Thermodynamics of Black Holes. Living Rev. Relativ. 2001, 4, 6. [CrossRef] [PubMed]

2. Landau, L.D.; Lifshitz, E.M. Statistical Physics; Elsevier: New York, NY, USA, 1980.

3. Pathria, R.K.; Beale, P.D. Statistical Mechanics; Butterworth-Heinemann: Oxford, UK, 2011.

4. Goodstein, D.L. States of Matter; Prentice-Hall: Englewood Cliffs, NJ, USA, 1975.

5. Sahay, A. Restricted thermodynamic fluctuations and the Ruppeiner geometry of black holes. Phys. Rev. D 2017, 95, 064002. [CrossRef]

6. Åman, J.E.; Bengtsson, I.; Pidokrajt, N. Thermodynamic Metrics and Black Hole Physics. Entropy 2015, 17, 6503-6518. [CrossRef]

7. Strominger, A.; Vafa, C. Microscopic origin of the Bekenstein-Hawking entropy. Phys. Lett. B 1996, $379,99$. [CrossRef]

8. Sen, A. Black hole entropy function, attractors and precision counting of microstates. Gen. Relativ. Gravit. 2008, 40, 2249. [CrossRef]

9. Benini, F.; Hristov, K.; Zaffaroni, A. Exact microstate counting for dyonic black holes in AdS 4 . Phys. Lett. B 2017, 771, 462 . [CrossRef]

10. Sarkar, T.; Sengupta, G.; Tiwari, B.N. Thermodynamic geometry and extremal black holes in string theory. J. High Energy Phys. 2008, 10, 076. [CrossRef]

11. Bellucci, S.; Tiwari, B.N. State-space correlations and stabilities. Phys. Rev. D 2010, 82, 084008 . [CrossRef]

12. Bellucci, S.; Tiwari, B.N. Thermodynamic Geometry and Topological Einstein-Yang-Mills Black Holes. Entropy 2012, 14, 1045. [CrossRef]

13. Wei, S.-W.; Liu, Y.-X. Insight into the Microscopic Structure of an AdS Black Hole from a Thermodynamical Phase Transition. Phys. Rev. Lett. 2015, 115, 111302. [CrossRef] [PubMed]

14. Chaturvedi, P.; Sengupta, G. Thermodynamic geometry and phase transitions of AdS braneworld black holes. Phys. Lett. B 2017, 765, 67. [CrossRef]

15. Sahay, A.; Sarkar, T.; Sengupta, G. On the phase structure and thermodynamic geometry of R-charged black holes. J. High Energy Phys. 2010, 11, 125. [CrossRef]

16. Chaturvedi, P.; Das, A.; Sengupta, G. Thermodynamic geometry and phase transitions of dyonic charged AdS black holes. Eur. Phys. J. C 2017, 77, 110. [CrossRef]

17. Gergely, L.; Pidokrajt, N.; Winitzki, S. Geometro-thermodynamics of tidal charged black holes. Eur. Phys. J. C 2011, 71, 1569. [CrossRef] 
18. Ruppeiner, G. Stability and fluctuations in black hole thermodynamics. Phys. Rev. D 2007, 75, 024037. [CrossRef]

19. Åman, J.E.; Bengtsson, I.; Pidokrajt, N. Geometry of Black Hole Thermodynamics. Gen. Relativ. Gravit. 2003, 35, 1733. [CrossRef]

20. Ruppeiner, G. Thermodynamics: A Riemannian geometric model. Phys. Rev. A 1979, 20, 1608. [CrossRef]

21. Ruppeiner, G. Riemannian geometry in thermodynamic fluctuation theory. Rev. Mod. Phys. 1995, 67, 605. [CrossRef]

22. Diósi, L.; Salamon, P. From Statistical Distances to Minimally Dissipative Processes. In Thermodynamics of Energy Conversion and Transport; Sieniutycz, S., De Vos, A., Eds.; Springer: New York, NY, USA, 2000.

23. Weinberg, S. Gravitation and Cosmology; Wiley: New York, NY, USA, 1972.

24. Ruppeiner, G. Thermodynamic curvature measures interactions. Am. J. Phys. 2010, 78, 1170. [CrossRef]

25. Ruppeiner, G.; Sahay, A.; Sarkar, T.; Sengupta, G. Thermodynamic geometry, phase transitions, and the Widom line. Phys. Rev. E 2012, 86, 052103. [CrossRef] [PubMed]

26. May, H.-O.; Mausbach, P. Riemannian geometry study of vapor-liquid phase equilibria and supercritical behavior of the Lennard-Jones fluid. Phys. Rev. E 2012, 85, 031201. [CrossRef] [PubMed]

27. Ruppeiner, G. Thermodynamic curvature from the critical point to the triple point. Phys. Rev. E 2012, 86, 021130. [CrossRef] [PubMed]

28. Widom, B. The critical point and scaling theory. Physica 1974, 73, 107. [CrossRef]

29. Ruppeiner, G. Riemannian geometric theory of critical phenomena. Phys. Rev. A 1991, 44, 3583. [CrossRef] [PubMed]

30. Ruppeiner, G. Riemannian geometric approach to critical points: General theory. Phys. Rev. E 1998, 57, 5135. [CrossRef]

31. Ruppeiner, G. Unitary Thermodynamics from Thermodynamic Geometry. J. Low Temp. Phys. 2014, 174, 13. [CrossRef]

32. Ruppeiner, G. Unitary Thermodynamics from Thermodynamic Geometry II: Fit to a Local-Density Approximation. J. Low Temp. Phys. 2015, 181, 77. [CrossRef]

33. Ruppeiner, G. Thermodynamic curvature and phase transitions in Kerr-Newman black holes. Phys. Rev. D 2008, 78, 024016. [CrossRef]

34. Ruppeiner, G. Equations of state of large gravitating gas clouds. Astrophys. J. 1996, 464, 547. [CrossRef]

35. Kaviani, K.; Dalafi-Rezaie, A. Pauli paramagnetic gas in the framework of Riemannian geometry. Phys. Rev. E 1999, 60, 3520. [CrossRef]

36. Ruppeiner, G. Riemannian geometry of thermodynamics and systems with repulsive power-law interactions. Phys. Rev. E 2005, 72, 016120. [CrossRef] [PubMed]

37. Ruppeiner, G. Partition function from no microscopics. Unpublished work, 2018.

38. Stanley, H.E. Scaling, universality, and renormalization: Three pillars of modern critical phenomena. Rev. Mod. Phys. 1999, 71, S358. [CrossRef]

39. Chamblin, A.; Emparan, R.; Johnson, C.V.; Myers, R.C. Charged AdS black holes and catastrophic holography. Phys. Rev. D 1999, 60, 064018. [CrossRef]

40. Åman, J.E.; Bengtsson, I.; Pidokrajt, N. Flat information geometries in black hole thermodynamics. Gen. Relativ. Gravit. 2006, 38, 1305. [CrossRef]

41. Davies, P.C.W. The Thermodynamic Theory of Black Holes. Proc. R. Soc. Lond. A 1977, 353, 499. [CrossRef]

42. Smarr, L. Mass Formula for Kerr Black Holes. Phys. Rev. Lett. 1973, 30, 71. [CrossRef]

43. Abbott, B.P.; Abbott, R.; Abbott, T.D.; Abernathy, M.R.; Acernese, F.; Ackley, K.; Adams, C.; Adams, T.; Addesso, P.; Adhikari, R.X.; et al. Observation of Gravitational Waves from a Binary Black Hole Merger. Phys. Rev. Lett. 2016, 116, 061102. [CrossRef] [PubMed]

44. Gezari, S.; Chornock, R.; Rest, A.; Huber, M.E.; Forster, K.; Berger, E.; Challis, P.J.; Neill, J.D.; Martin, D.C.; Heckman, T.; et al. An ultraviolet-optical flare from the tidal disruption of a helium-rich stellar core. Nature 2012, 485, 217. [CrossRef] [PubMed]

45. Janyszek, H.; Mrugała, R. Riemannian geometry and stability of ideal quantum gases. J. Phys. A Math. Gen. 1990, 23, 467. [CrossRef] 
46. Oshima, H.; Obata, T.; Hara, H. Riemann scalar curvature of ideal quantum gasses obeying Gentile's statistics. J. Phys. A Math. Gen. 1999, 32, 6373. [CrossRef]

47. Quevedo, H. Geometrothermodynamics of black holes. Gen. Relativ. Gravit. 2008, 40, 971. [CrossRef]

48. Shen, J.; Cai, R.-G.; Wang, B.; Su, R.-K. Thermodynamic geometry and critical behavior of black holes. Int. J. Mod. Phys. A 2007, 22, 11. [CrossRef]

49. Sahay, A.; Sarkar, T.; Sengupta, G. On the thermodynamic geometry and critical phenomena of AdS black holes. J. High Energy Phys. 2010, 7, 082. [CrossRef]

50. Niu, C.; Tian, Y.; Wu, X.-N. Critical phenomena and thermodynamic geometry of Reissner-Nordström-anti-de Sitter black holes. Phys. Rev. D 2012, 85, 024017. [CrossRef]

51. Cvetič, M.; Nojiri, S.; Odintsov, S.D. Black hole thermodynamics and negative entropy in de Sitter and anti-de Sitter Einstein-Gauss-Bonnet gravity. Nucl. Phys. B 2002, 628, 295. [CrossRef]

52. Nojiri, S.; Odintsov, S.D. Regular multihorizon black holes in modified gravity with nonlinear electrodynamics. Phys. Rev. D 2017, 96, 104008. [CrossRef]

53. Clunan, T.; Ross, S.F.; Smith, D.J. On Gauss-Bonnet black hole entropy. Class. Quantum Grav. 2004, $21,3447$. [CrossRef]

54. Sahay, A.; Jha, R. Geometry of criticality, supercriticality, and Hawking-Page transitions in Gauss-Bonnet-AdS black holes. Phys. Rev. D 2017, 96, 126017. [CrossRef]

55. Åman, J.E.; Pidokrajt, N. Geometry of higher-dimensional black hole thermodynamics. Phys. Rev. D 2006, 73, 024017. [CrossRef]

56. Åman, J.E.; Pidokrajt, N. On explicit thermodynamic functions and extremal limits of Myers-Perry black holes. Eur. Phys. J. C 2013, 73, 2601. [CrossRef]

57. Zhang, J.-L.; Cai, R.-G.; Yua, H. Phase transition and thermodynamical geometry for Schwarzschild AdS black hole in $\mathrm{AdS}_{5} \times \mathrm{S}^{5}$ spacetime. J. High Energy Phys. 2015, 2, 143. [CrossRef]

58. Arcioni, G.; Lozano-Tellechea, E. Stability and critical phenomena of black holes and black rings. Phys. Rev. D 2005, 72, 104021. [CrossRef]

59. Mirza, B.; Zamaninasab, M. Ruppeiner geometry of RN black holes: flat or curved? J. High Energy Phys. 2007, 6, 059. [CrossRef]

60. Sahay, A.; Sarkar, T.; Sengupta, G. Thermodynamic geometry and phase transitions in Kerr-Newman-AdS black holes. J. High Energy Phys. 2010, 4, 118. [CrossRef]

61. Kubizn̆ák, D.; Mann, R.B. P-V criticality of charged AdS black holes. J. High Energy Phys. 2012, 7, 033. [CrossRef]

62. Dolan, B.P. Intrinsic curvature of thermodynamic potentials for black holes with critical points. Phys. Rev. D 2015, 92, 044013. [CrossRef]

63. Wei, S.-W.; Liu, Y.-X. Critical phenomena and thermodynamic geometry of charged Gauss-Bonnet AdS black holes. Phys. Rev. D 2013, 87, 044014. [CrossRef]

64. Wei, S.-W.; Liu, Y.-X.; Wang, Y.-Q.; Guo, H. Thermodynamic geometry of black hole in the deformed Hořava-Lifshitz gravity. EPL Eur. Lett. 2012, 99, 20004. [CrossRef]

65. Bagher, M.; Poshteh, J.; Riazi, N. Phase transition and thermodynamic stability in extended phase space and charged Hořava-Lifshitz black holes. Gen. Relativ. Gravit. 2017, 49, 64.

66. Deng, G.-M.; Huang, Y.-C. $Q-\Phi$ criticality and microstructure of charged AdS black holes in $f(R)$ gravity. Int. J. Mod. Phys. A 2017, 32, 1750204. [CrossRef]

67. Li, G.-Q.; Mo, J.-X. Phase transition and thermodynamic geometry of $f(R)$ AdS black holes in the grand canonical ensemble. Phys. Rev. D 2016, 93, 124021. [CrossRef]

68. Zangeneh, M.K.; Dehyadegari, A.; Mehdizadeh, M.R.; Wang, B.; Sheykhi, A. Thermodynamics, phase transitions and Ruppeiner geometry for Einstein-dilaton-Lifshitz black holes in the presence of Maxwell and Born-Infeld electrodynamics. Eur. Phys. J. C 2017, 77, 423. [CrossRef]

69. Wei, Y.H. Thermodynamic Properties of a Regular Black Hole in Gravity Coupling to Nonlinear Electrodynamics. Entropy 2018, 20, 192. [CrossRef]

70. Banerjee, R.; Modak, S.K.; Roychowdhury, D. A unified picture of phase transition: from liquid-vapour systems to AdS black holes. J. High Energy Phys. 2012, 10, 125. [CrossRef] 
71. Lala, A.; Roychowdhury, D. Ehrenfest's scheme and thermodynamic geometry in Born-Infeld AdS black holes. Phys. Rev. D 2012, 86, 084027. [CrossRef]

72. Hendi, S.H.; Talezadeh, M.S.; Armanfard, Z. Phase Transition of Black Holes in Brans-Dicke Born-Infeld Gravity through Geometrical Thermodynamics. Adv. High Energy Phys. 2017, 2017, 7158697. [CrossRef] 